\title{
Spin polarization of Auger- and of photoelectrons from barium atoms exposed to circularly polarized radiation and their cross comparison
}

\author{
R. Kuntze, M. Salzmann, N. Böwering, U. Heinzmann \\ Fakultät für Physik, Universität Bielefeld, Universitätsstrasse 25, D-33615 Bielefeld, Germany, \\ and Fritz-Haber-Institut der Max-Planck-Gesellschaft, D-14195 Berlin, Germany
}

Received: 16 July 1993/Final version: 24 August 1993

\begin{abstract}
New results of spin polarization of both photoelectrons and Auger electrons are reported after $5 p$ photoionization of free $\mathrm{Ba}$ atoms with circularly polarized light. A substantial polarization transfer from the spin polarized photons to the spin polarized photoelectrons and via the hole state orientation to the spin polarized Auger-electrons is observed. The cross comparison of the results for photoelectrons and Auger-electrons allows a quantitative test of the assumed two step model where both electron-emission processes occur in sequence.
\end{abstract}

PACS: $32.80 . \mathrm{Fb} ; 32.80 . \mathrm{Hd}$

Creation of vacancies in inner atomic shells by photon or particle impact often gives rise to subsequent Auger decay. Recently, these Auger electrons have been measured to be strongly spin polarized for the electron spectroscopy studies with free barium atoms [1] and $\mathrm{Rb}$-adsorbates [2], where the primary photoemission process has been performed by means of spin polarized i.e. circularly polarized radiation. In their emission characteristics these Auger electrons reflect a non-statistical population of the magnetic substates of the intermediate ionic state produced by the spin oriented initial impact $[3,4]$. Consequently, an orientation of the intermediate state can be transferred partially to the Auger electron spin polarization $[5,6]$. On the other hand the data of spinand angle resolved Auger spectroscopy can be used to determine alignment and orientation of the intermediate photoion state from the experiment [1]. Cross comparison of these results with the corresponding dynamical parameters characterizing the primary photoelectron emission process, angular distribution and spin polarization parameters which form a "complete experiment", should answer whether photoelectron and Auger electron emission are subsequent processes in a two-step model or whether they have to be seen as a dynamical combi- nation. A mutual influence of Auger- and photoelectrons would give rise to the so-called post-collision interaction, indicating a break-down of the two-step model. A reference case is the $4 d$ ionization of xenon [7] for which both the occurrence [8] and the vanishing [9] of postcollision interaction was discussed in detail.

Figure 1 shows the angle-resolved measurement [1] of the spin-polarization component $A(\Theta)$ (parallel to the photon beam) of Auger electrons in the $O_{3} P_{1} P_{1}\left({ }^{1} S_{0}\right)$ Auger line of $\mathrm{Ba}$ atoms in photoionization using circularly polarized synchrotron radiation from the $6.5 \mathrm{~m}$ N.I. Monochromator ( $\Delta \lambda=0.18 \mathrm{~nm}$ FWHM) at BESSY [10]. For Auger electrons the angular distribution of the polarization component $A(\Theta)$ has the same analytical form as for photoelectrons [10]:

$A(\Theta)=\gamma \frac{A-\alpha P_{2}(\cos \Theta)}{1-(\beta / 2) \cdot P_{2}(\cos \Theta)}$

with the second Legendre polynomial $P_{2}(\cos \Theta)$ and the light helicity $\gamma$ but with different dynamical parameters for the angular distribution of the spin polarization. Since the photoelectrons in the example [1] of Fig. 1 were too slow $(<1 \mathrm{eV})$ to be analyzed, we had to repeat the experiment at photon energies higher than $23.5 \mathrm{eV}$ in order

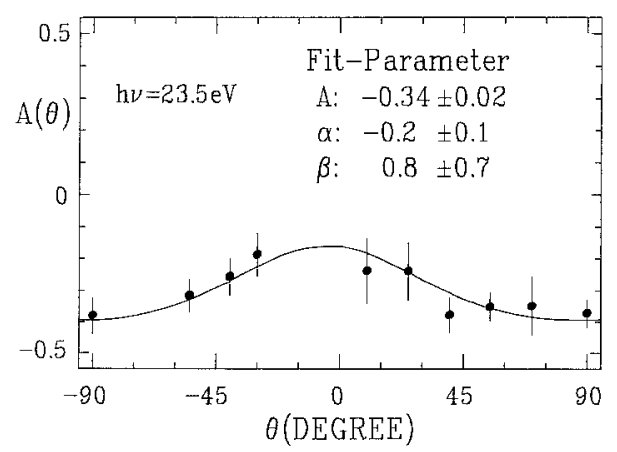

Fig. 1. Measured angular dependence of the Auger electron spin polarization at $h v=23.5 \mathrm{eV}$ for the Auger electrons with $7.5 \mathrm{eV}$ kinetic energy corresponding to a $p_{\frac{3}{2}}^{-1}$ hole state [1] 
to include the simultaneous detection of the corresponding photoelectrons and their spin polarization. It is the purpose of this publication to present these data obtained with circularly polarized photons at energies around $28 \mathrm{eV}$ and to compare the spin polarization values of Augerand photoelectrons measured.

The ionization process of interest can be denoted by:

$$
\begin{aligned}
\mathrm{Ba} & \left(5 p^{6} 6 s^{2}\right){ }^{1} S_{0}+h v \\
& \rightarrow \mathrm{Ba}^{+}\left(5 p^{5} 6 s^{2}\right)^{2} P_{J}+e_{\text {Photo }}^{-}(\varepsilon s, \varepsilon d) \\
& \rightarrow \mathrm{Ba}^{2+}\left(5 p^{6}\right)^{1} S_{0}+e_{\text {Photo }}^{-}+e_{\text {Auger }}^{-}(\varepsilon p)
\end{aligned}
$$

where $J=\frac{3}{2}$ or $\frac{1}{2}$ is the total angular momentum of the photoion. For the $J=\frac{1}{2}$ channel the photoelectrons leave the ion either in the $\varepsilon s_{\frac{1}{2}}$ or the $\varepsilon d_{\frac{3}{2}}$ continuum state with a spin polarization transfer, characterized by the dynamical parameter $A$, determined only by the ratio $\lambda=$ $\left|D_{s} / D_{d}\right|$ of reduced matrix elements as being [11]:

$$
A_{\frac{1}{2}}^{\text {photo }}=-\frac{1}{2} \frac{1-2 \lambda^{2}}{1+\lambda^{2}} \text {. }
$$

In the $J=\frac{3}{2}$ channel a phase shift difference between the outgoing $d_{\frac{5}{2}}$ and $d_{\frac{3}{2}}$ partial wave, and different $D_{d_{\frac{5}{2}}, \frac{3}{2}}$ matrix elements have to be taken into account in the photoelectron spin polarization $A_{\frac{3}{2}}$. In the non-relativistic approximation only, neglecting any influence of the spin orbit interaction in the continuum states [12],

$$
A_{\frac{3}{2}}^{\text {photo }}=\frac{1}{4} \frac{1-2 \lambda^{2}}{1+\lambda^{2}}=-\frac{1}{2} A_{\frac{1}{2}}^{\text {photo }}
$$

is also only a function of the ratio of the matrix elements $\lambda$.

Assuming that the photoemission and the Auger process occur in sequence and that the transition probability of one process is not influenced by the other, the Auger electron spin polarization $A_{\frac{3}{2}, \frac{1}{2}}^{\text {Auger }}$ can be factorized [6] as being the product of the Auger decay parameter $\beta_{1}$ and the photoion orientation, which is proportional to the photoelectron spin polarization $A_{\frac{3}{2}, \frac{1}{2}}^{\text {photo }}$ in the nonrelativistic limit. According to the theory of Kabachnik and Lee [6] this is given for the above mentioned Auger decay of $\mathrm{Ba}$ to be

$$
\begin{aligned}
& A_{\frac{1}{2}}^{\text {Auger }}=-\frac{1}{3} A_{\frac{1}{2}}^{\text {photo }} \\
& A_{\frac{3}{2}}^{\text {Auger }}=-\frac{5}{3} A_{\frac{3}{2}}^{\text {photo }} .
\end{aligned}
$$

The second equation is only valid in the non-relativistic limit, neglecting any differences between the $\varepsilon d_{\frac{3}{2}}$ and $\varepsilon d_{\frac{5}{2}}$ continuum waves of photoelectrons [13] due to spin orbit interaction. In the relativistic case [14] the Auger electron polarization depends upon two ratios of matrix elements whereas the photoelectron polarization depends in addition on a phase shift difference and therefore no linear relation exists between $A^{\text {Auger }}$ and $A^{\text {photo }}$.

Besides the spin polarization transfer from spin-polarized photons to spin polarized photoelectrons and via the orientation of the hole states to spin polarized Auger electrons, there exists also a dynamic spin polarization of electrons (Auger [15] as well as photoelectrons [11]) due to the spin orbit interaction and phase shift differences between at least two outgoing partial continuum waves.

This polarization component is perpendicular to the reaction plane, given by the momenta of incoming radiation and of outgoing detected electrons, and is characterized by the dynamical spin parameter $\xi$

$$
P_{\perp}^{\text {Auger, photo }}=\xi^{\text {Auger, photo }} \frac{2 \sin \Theta \cos \Theta}{1-\frac{\beta^{\text {Auger, photo }}}{2} \cdot P_{2}(\cos \Theta)}
$$

with $\beta$ denoting the asymmetry parameter of the corresponding angular electron intensity distribution. For a single channel Auger decay $\xi^{\text {Auger }}$ should vanish for the above-mentioned Auger transition, since there is only one continuum state $(\varepsilon p)$ which cannot interfere with itself. Thus a deviation from $\xi^{\text {Auger }} \equiv 0$ would also be an indication of the invalidity of the two-step-model.

Figure 2 shows on the left side the experimental results of the spin polarization parameter $A$ (full points) and $\xi$ (open points) measured for the photoelectrons leaving behind the ion in the ${ }^{2} P_{\frac{1}{2}}$ or ${ }^{2} P_{\frac{3}{2}}$ ionic state. The right side of Fig. 2 gives the corresponding Auger electron polarizations measured. The middle part of Fig. 2 shows the Auger electron polarization expected and calculated on the basis of the experimental photoelectron polarization data (left part of Fig. 2) within the model of the two-step process and within the LS-approximation (non-rela-
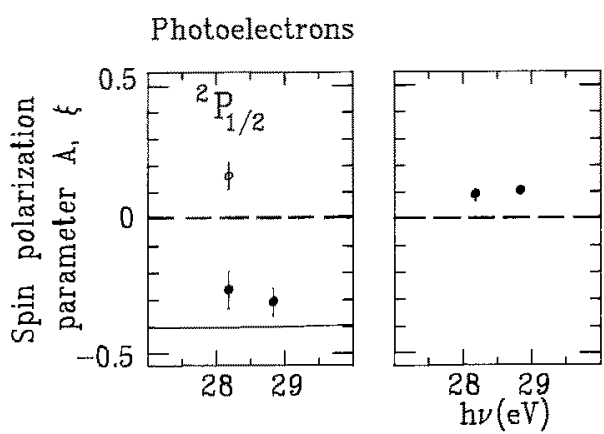

Augerelectrons
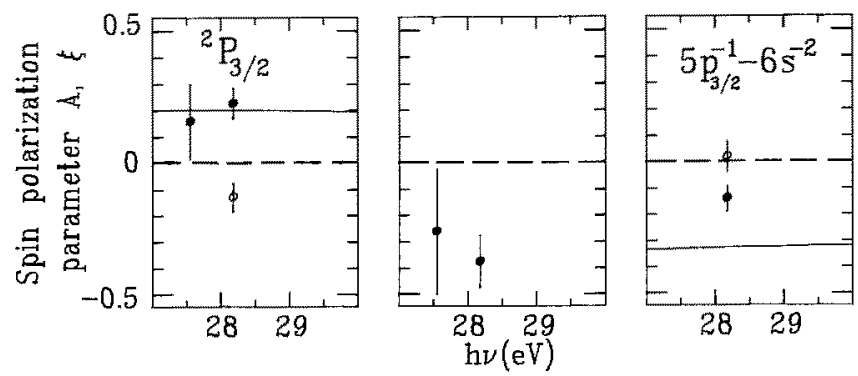

Fig. 2. Spin polarization parameters $A$ (full points) and $\xi$ (open points) measured for photoelectrons (left part) and Auger electrons (right part) in comparison with theoretical HF predictions [17] for $A$ (full curves) and with Auger electron predictions (middle part) based upon the experimental values of the photoelectrons (left part). ${ }^{2} P_{\frac{1}{2}}$ and ${ }^{2} P_{\frac{3}{2}}$ denote the intermediate ionic states 
tivistic model) for the channel of the ${ }^{2} P_{\frac{3}{2}}$ ion. It is worth noting that this last LS restriction does not apply for the ${ }^{2} P_{\frac{1}{2}}$ ionic channel since $A^{\text {Auger }}$ and $A^{\text {photo }}$ are proportional to each other. Finally, Fig. 2 left and right parts show a comparison with theoretical predictions based upon matrix elements obtained by means of Hartree-Fock calculations [17] (full curves). Since for both ionic channels ${ }^{2} P_{\frac{1}{2}}$ and ${ }^{2} P_{\frac{3}{2}}$ the Auger electron spin parameter $\xi$ has been measured to be $-0.06 \pm 0.08$ and $0.02 \pm 0.06$, respectively, (close to zero within the experimental uncertainty), there is no indication of the invalidity of the two-step model used. The ratio of $A_{\frac{1}{2}}^{\text {photo }} / A_{\frac{3}{2}}^{\text {photo }}$ as well as of $\xi_{\frac{1}{2}}^{\text {photo }} / \xi_{\frac{3}{2}}^{\text {photo }}$ is not -2 as predicted for the non-relativistic treatment. This could be due to a strong influence of the spin-orbit interaction onto the $\varepsilon d_{\frac{3}{2}}$ and $\varepsilon d_{\frac{5}{2}}$ continuum states in the $5 p \rightarrow \varepsilon d$ photoionization process or due to the strong $5 d-6 s$ configuration interaction for the $\mathrm{Ba}^{+}$state with the $5 p_{\frac{3}{2}}-$ hole [18]. Thus the corresponding Auger electron polarizations calculated by means of the photoelectron polarizations and given in the middle part of Fig. 2 do not need to agree with the directly measured $A_{\frac{3}{2}}^{\text {Auger }}$ value given in the right part.

This cross comparison can, however, be made for the ${ }^{2} P_{1}$ channel, where the photoelectron polarization is not influenced by any phase shift differences and thus directly connected to the Auger electron polarization via the hole orientation. Since this comparison between the calculated (middle part) and directly measured (right part) $A_{\frac{1}{2}}^{\text {Auger }}$ results shows agreement within the experimental uncertainties, it might be summarized that the results reported in this paper do not disagree with the two-step model assuming the emissions of photoelectron and Auger elec- tron to occur in sequence without significant post-collision interaction.

Financial support by the BMFT (055 PBAXI) is gratefully acknowledged.

\section{References}

1. Kuntze, R., Salzmann, M., Böwering, N., Heinzmann, U.: Phys. Rev. Lett. 70, 3716 (1993)

2. Stoppmanns, P., Schmiedeskamp, B., Vogt, B., Müller, N., Heinzmann, U.: Phys. Scr. T41, 190 (1992)

3. Flügge, S., Mehlhorn, W., Schmidt, V.: Phys. Rev. Lett. 29, 7 (1972)

4. Mehlhorn, W.: In: Atomic inner shell physics. Crasemann, B. (ed.), p. 119. New York: Plenum 1985

5. Klar, H.: J. Phys. B13, 4741 (1980)

6. Kabachnik, N.M., Lee, O.V.: J. Phys. B22, 2705 (1989)

7. Schmidt, V.: Z. Phys. D2, 275 (1986); J. Phys. (Paris) Colloq. 48, C9-401 (1987)

8. Schmidt, V., Krummacher, S., Wuilleumier, F.J., Dhez, P.: Phys. Rev. A24, 1803 (1981)

9. Borst, M., Schmidt, V.: Phys. Rev. A33, 4456 (1986)

10. Heckenkamp, Ch., Schäfers, F., Schönhense, G., Heinzmann, U.: Phys. Rev. Lett. 52, 421 (1984)

11. Heinzmann, U.: J. Phys. B13, 4353, 4367 (1980)

12. Cherepkov, N.A.: Sov. Phys.-JETP 38, 463 (1974)

13. Heckenkamp, Ch., Schäfers, F., Schönhense, G., Heinzmann, U.: Z. Phys, D2, 257 (1986)

14. Bußert, W., Klar, H.: Z. Phys. A312, 315 (1983)

15. Hahn, U., Semke, J., Merz, H., Kessler, J.: J. Phys. B 18, L417 (1985)

16. Kabachnik, N.M., Sazhina, I.P.: J. Phys. B23, L353 (1990)

17. Berezhko, E.G., Ivanov, V.K., Kabachnik, N.M.: Phys. Lett. A 66, 474 (1978)

18. Bizau, J.M., Cubaynes, D., Gérard, P., Wuilleumier, F.: Phys. Rev. A40,3002 (1989) 